\title{
Implementation of robot-assisted stereoelectroencephalography for resective epilepsy surgery
}

\author{
C. Teixell, S. Pacreu, J. Fernandez, L. Moltó, L. Serrano, E. Vila \\ Hospital Parc de Salut Mar, Barcelona
}

\section{Background}

Robotic stereoelectroencephalography (SEEG) is a method that allows getting precise information from deep cortical areas through the implantation of deep electrodes, avoiding the need of craniotomies. It has a clear application in patients with drug-resistant focal epilepsy, as it defines anatomically the epileptogenic zone.

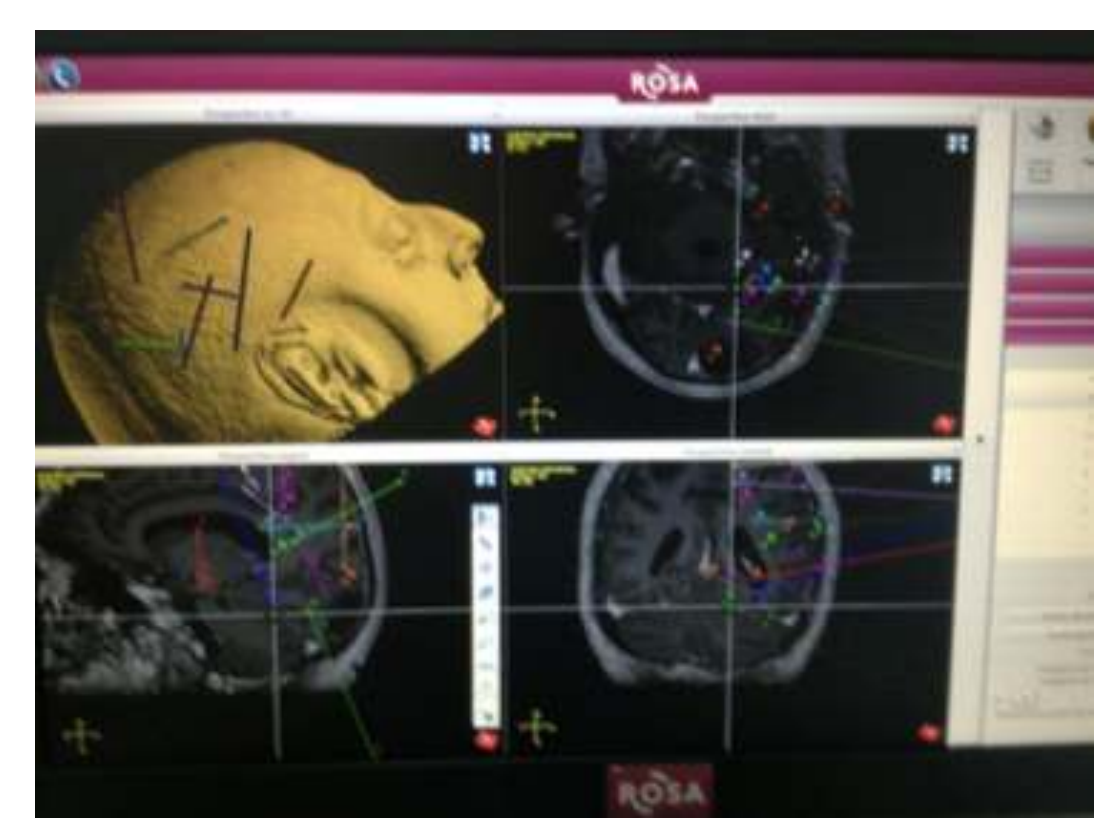

\section{Objective}

To analyse the current perioperative management and the incidence of complications during this procedure.

\section{Material and methods}

We collected retrospectively 30 cases of patients with medically refractory focal epilepsy who underwent robotic stereotactic placement of deep electrodes between January 2013 and March 2016.

\section{Results and discussion}

Nineteen patients had no other pathologies but epilepsy. The average number of electrodes placed was 11. We used standard monitorization for all cases, and in fourteen of them invasive blood pressure was also measured. There were no severe complications during the surgery, just two to remark: arterial bleeding with the placement of an electrode and short delay on awakening. Both cases were solved without consequences.

As soon as it was possible the patients were discharged in order to record seizures and functional brain mapping.

Recent meta-analysis estimates a 1-4\% incidence of complications during SEEG $(2,3)$. We registered three severe complications: an acute subdural hematoma that required craniotomy, an intraparenchymal hematoma that cursed with mild aphasia and a brain abscess that required drainage.

\section{Conclusions}

SEEG is an accurate technique and useful to delimit the epileptogenic zone with a promising future. However, we must not underestimate the possible complications. Establishing protocols can help us in the handling of these patients. In our particular experience, after observing these results we changed our protocol in two main factors: decreasing the number of electrodes and performing a brain CT before awakening the patient. 